\title{
Predictors of loss to follow-up before HIV treatment initiation in Northwest Ethiopia: a case control study
}

\author{
Ismael Ahmed ${ }^{1 *}$, Salem T Gugsa ${ }^{2}$, Seblewengel Lemma ${ }^{3}$ and Meaza Demissie ${ }^{3}$
}

\begin{abstract}
Background: In Ethiopia, there is a growing concern about the increasing rates of loss to follow-up (LTFU) in HIV programs among people waiting to start HIV treatment. Unlike other African countries, there is little information about the factors associated with LTFU among pre-antiretroviral treatment (pre-ART) patients in Ethiopia. We conducted a case-control study to investigate factors associated with pre-ART LTFU in Ethiopia.

Methods: Charts of HIV patients newly enrolled in HIV care at Gondar University Hospital (GUH) between September 11, 2008 and May 8, 2011 were reviewed. Patients who were "loss to follow-up" during the pre-ART period were considered to be cases and patients who were "in care" during the pre-ART period were controls. Logistic regression analysis was used to explore factors associated with pre-ART LTFU.

Results: In multivariable analyses, the following factors were found to be independently associated with pre-ART LTFU: male gender [Adjusted Odds Ratio (AOR) = 2.00 (95\% Cl: 1.15, 3.46)], higher baseline CD4 cell count (251-300 cells $/ \mu \mathrm{l}[\mathrm{AOR}=2.64(95 \% \mathrm{Cl}: 1.05,6.65)], 301-350 \mathrm{cells} / \mu \mathrm{l}[\mathrm{AOR}=5.21(95 \% \mathrm{Cl}: 1.94,13.99)]$, and $>350 \mathrm{cells} / \mu \mathrm{l}$ $[A O R=12.10(95 \% \mathrm{Cl}: 6.33,23.12)]$ compared to CD4 cell count of $\leq 200 \mathrm{cell} / \mathrm{s} / \mathrm{\mu l})$ and less advanced disease stage (WHO stage I $[\mathrm{AOR}=2.81(95 \% \mathrm{Cl}: 1.15,6.91)]$ compared to WHO stage IV). Married patients $[\mathrm{AOR}=0.39$ (95\% Cl: 0.19, 0.79)] had reduced odds of being LTFU. In addition, patients whose next visit date was not documented on their medical chart $[A O R=241.39$ (95\% Cl: 119.90, 485.97)] were more likely to be LTFU.

Conclusion: Our study identified various factors associated with pre-ART LTFU. The findings highlight the importance of giving considerable attention to pre-ART patients' care from the time that they learn of their positive HIV serostatus. The completeness of the medical records, the standard of record keeping and obstacles to retrieving charts also indicate a serious problem that needs due attention from clinicians and data personnel.
\end{abstract}

Keywords: Pre-antiretroviral treatment loss to follow-up, HIV patients, Case control, Ethiopia, Africa

\section{Background}

Sub-Saharan Africa bears the lion's share of the global Human Immunodeficiency Virus (HIV) burden, with 22.5 million infected people, which constitutes $68 \%$ of the global total [1]. According to the Ethiopian HIV related estimates and projections for the year 2012, there are759268 People Living with HIV/AIDS (PLHIV) in Ethiopia which is $1.3 \%$ of the national adult HIV

\footnotetext{
* Correspondence: ismaelahmed2003@gmail.com

'University of Gondar and Addis Continental Institute of Public Health, P.O. Box 180432, Addis Ababa, Ethiopia

Full list of author information is available at the end of the article
}

prevalence. The Amhara region, which is one of the nine regional states of Ethiopia located in the northern part of the country, carries $27 \%$ of the national HIV burden with an estimated 1.4\% adult HIV prevalence [2]. Ethiopia introduced a fee based Anti-Retroviral Treatment (ART) program in 2003. Since 2005, much progress has been made in making free ART available to a large number of Ethiopians [3]. By the end of June 2011, out of 580909 PLHIV who had been enrolled in chronic care, 333434 (57\%) patients had started ART but only 247805 (74\%) were currently on treatment [4].

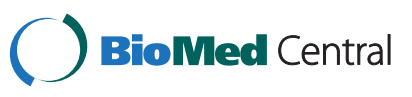


There is a growing concern about the increasing rates of LTFU in HIV programs among people already on treatment and those waiting to start HIV treatment. Studies from Ethiopia, South Africa, Uganda, and Cambodia have demonstrated that there is a high rate of pre-ART mortality and LTFU [5-9]. However, compared to patients who have started ART, less emphasis has been given to the follow-up of pre-ART patients, making them a neglected population $[5,10,11]$. Unlike the follow-up of PLHIV who are taking ART, there is no standardized definition of LTFU and appointment system for pre-ART patients and this contributes to the challenges of tracing those LTFU in Ethiopia [5]. This gap highlights the need for greater focus upon retention of pre-ART patients from the beginning of HIV care, not just after patients have been initiated on ART. In Ethiopia, there is little information about the magnitude of the problem or the associated factors that contribute to LTFU among pre-ART patients. Therefore, this study set out to identify factors associated with preART LTFU among newly enrolled HIV-infected patients.

\section{Methods \\ Study design}

The study design was a case-control study using chart review of HIV patients newly enrolled in pre-ART care retrospectively between September 11, 2008 and May 8, 2011.

\section{Study setting}

The study was conducted in Gondar University Hospital, Gondar town in the Amhara region of Ethiopia, about 735 kilometers north of the capital city Addis Ababa. The hospital has 400 beds and serves more than five million people. The hospital began ART service provision in March 2005. In August 2007, the hospital started HIV/ AIDS case management program by deploying trained adherence case managers and adherence supporters to assess patients' needs, develop patient-centered care plan, and provide adherence counseling, health education, psychosocial support, trace patients who are LTFU, monitor patients "at-risk for non-adherence" and link patients to community resources [12]. According to the Ethiopian guideline for the implementation of HIV/AIDS case management, adherence case managers are trained nonhealth care professionals who are high school graduate with some experience on HIV/AIDS. Adherence supporters are PLHIV with a minimum of an $8^{\text {th }}$ grade education that are enrolled in HIV care and demonstrated good adherence to HIV care. The guideline sets clinical and social criteria including poor adherence, history of other chronic illnesses, history of substance abuse, problems of accessing food, lack of shelter, lack of psychosocial support, etc. to screen and identify "at-risk for non-adherence" patients or patients who may likely not adhere to treatment and care and potentially become LTFU [3].
Patients are linked to the HIV clinic either from HIV testing clinics within the hospital or formally referred (transferred in) by other health facilities. At presentation to the clinics, all patients undergo a complete assessment using standardized intake forms whereby a full clinical history and examination is conducted to determine WHO HIV disease staging and screen for the presence of opportunistic diseases. According to the national guideline, HIV-positive adults and adolescents are considered eligible for ART if they are in WHO clinical stage IV (irrespective of CD4 count), or in stage III if the CD4 cell count is $\leq 350$ cells $/ \mu \mathrm{l}$, or in any of the WHO clinical stages if the CD4 cell count is $<200$ cells $/ \mu \mathrm{l}$. ART is also recommended for all patients with active pulmonary tuberculosis (TB) with CD4 cell count $<350$ cells $/ \mu$ l, or patients with extra-pulmonary or disseminated TB irrespective of their CD4 cell count. If CD4 cell count measurement is not available, all patients with active TB are eligible for ART. Noneligible patients are provided with an appointment to attend the hospital every three months for cotrimoxazole preventive therapy (CPT) and every six months for laboratory evaluation (including measurement of CD4 cell count) [13].

In GUH, patients who are eligible for ART receive preliminary counseling and then are asked to return home with a cotrimoxazole prescription (if applicable) and prepare for ART initiation within two weeks. They are requested to come back to the hospital accompanied by a relative, if possible, to foster a supportive home environment and reinforce the importance of treatment adherence. All new HIV positive patients who are enrolled into chronic HIV care are linked to adherence case managers for counseling and education on HIV infection and the importance of adherence to care. The adherence case managers also conduct a needs assessment to identify patients who are "at-risk for nonadherence" [3]. There is a tracking system in place to trace patients who are LTFU during the pre-ART and ART periods. However, unlike ART patients who are labeled as "lost" when patients have not seen for more than one month and "dropped" when having been loss to follow-up for over three months, there is no standardized definition of LTFU being used for preART patients. This makes it a challenge to track pre-ART patients and identify LTFU in a timely fashion [5]. By the end of June 2011, a total of 9236 HIV patients had been enrolled in HIV chronic care service and 6178 had been initiated on ART. At the time of the study, the ART clinic was equipped with a total of 27 trained staff, including one physician, one health officer, eight nurses, two pharmacy technicians, three adherence case managers, eight adherence supporters, and four data personnel. 


\section{Study participants}

The study population was those HIV positive patients who were newly enrolled and followed up in the preART care at GUH from September 11, 2008 to May 8, 2011. HIV patients who were aged $<15$ years old, pregnant, transferred in from another health facility, transferred out to another health facility and known to have died before starting ART were excluded from the study. In addition to this, those patients who started ART within the first two weeks following pre-ART enrollment were excluded from the study due to the short pre-ART follow-up period.

\section{Variables}

In this study, the explanatory variables were sociodemographic and clinical variables including age, gender, marital status, level of education, religion, residence, patient referral information, employment status, HIV disclosure status, history of $\mathrm{TB}$, baseline $\mathrm{CD} 4$ count, and WHO staging and the dependent variable was pre-ART LTFU. The major pre-ART outcome was defined and analyzed as 'pre-ART loss to follow-up' or 'in care'.

\section{Definitions}

We defined 'Pre-ART loss to follow-up' (cases) to include patients who were not on ART and did not return for care for a period of one month or more since their most recent documented appointment date, $[5,14,15]$. For patients whose next appointment date was not documented, 'pre-ART loss to follow-up' included patients who were not on ART and did not return to care for seven months or more since their last hospital visit.

Patients who were 'in care' (controls) were defined as patients who were alive, followed pre-ART care as scheduled [16], visited the hospital within seven months of their last visit, or were currently on ART.

\section{Measurement}

The data sources were the Federal Ministry of Health patients' registration database and intake forms which are completed during the enrollment of HIV patients in chronic care. The data abstraction tool for chart review was prepared based on the information contained within the patient registration and follow-up card, including the socio-demographic and clinical variables of interest. Once eligible patients for the study were identified based on the inclusion criteria, study data was abstracted by four experienced ART prescriber nurses.

Patients' length of follow-up time in pre-ART care was grouped in to less than one month, one to six months, seven to 12 months and greaterthan 12 months for analysis. Accordingly, LTFU patients who only had the first visit to HIV clinic were categorized under less than one month follow-up time in pre-ART care.

\section{Study size}

The sample size was calculated using the following assumptions for an unmatched case-control study design: $95 \%$ confidence interval $(\mathrm{CI}), 80 \%$ power, $25 \%$ level of exposure in the 'in care' (control) group, 1:2 ratio of casesto-controls, and 1.5 expected odds ratio. According to the data from GUH HIV clinic, about 3000 PLHIV were enrolled in pre-ART care in the period of September 11, 2008 to May 8, 2011, amounting to 32 months of cohort data to be studied. After identifying the list of all eligible study subjects, cases and controls were listed separately and simple random sampling technique was used to select the sample cases and controls using SPSS version 18 .

\section{Data analysis}

Data coding, entry and cleaning was carried out using Epi Info version 3.5.1 and the analysis was carried out using SPSS version 18. Univariate analysis was carried out to describe baseline socio-demographic and clinical characteristics of the study subjects using simple frequency distribution. Chi-squared tests were used to measure the significance of differences in socio-demographic and clinical characteristic between cases and controls. Confounders were controlled for during the analysis stage using logistic regression and computed adjusted odds ratios with 95\% confidence intervals. We have also checked for interaction between variables using the logistic regression after creating the interaction terms.

Charts with incomplete data were entered as "missing" and the rate of missing data for different variables was below $5 \%$ except the employment status variable which was excluded without replacement from the logistic regression analysis.

\section{Ethical approval}

Ethical clearance was obtained from the institutional review board of the University of Gondar and Addis Continental Institute of Public Health. Confidentiality of patients' data was ensured. While reviewing patients' records, non-personal identifiers such as patients' medical registration number and pre-ART number were used to distinguish study subjects during data collection. We communicated the list of LTFU patients to adherence supporters for future tracing to establish their current status and assist them to resume their care.

\section{Results}

Between September 11, 2008 and May 8, 2011, a total of 3035 (1240 males and 1795 females) HIV patients were newly enrolled for HIV care at GUH ART clinic. Of these, 1979 were eligible for this study based on the inclusion criteria. Of the eligible patients, $8 \%(n=162)$ of pre-ART patients were excluded from the study because 
their charts could not be found. Out of the remaining 1817 study population, $70.8 \%(\mathrm{n}=1287)$ were patients who were under active follow-up for their HIV care while the other $29.2 \%(n=530)$ were lost to follow-up during the pre-ART period (Figure 1). In this study, a total of 1089 (363 cases and 726 controls) patient charts were reviewed from 1817 study population.

\section{Baseline socio-demographic characteristics of the study population}

Out of the 1089 study subjects analyzed, 59.7\% (650) were female. The majority $(59.5 \% ; n=316)$ of patients who were pre-ART LTFU were also females. By the time of enrollment into HIV care, nearly half $(45.7 \%$; $n=498)$ of the study subjects were between 25-34 years of age. The mean age distribution was 32.7 years with a standard deviation of 9.12. Of the total study population, $45.7 \%(\mathrm{n}=498)$ were married. With regards to educational status of study subjects, $38.3 \%(n=417)$ of patients completed secondary school or above followed by $35.3 \%$ $(\mathrm{n}=384)$ of patients with no education. The majority $(91 \% ; \mathrm{n}=991)$ of study subjects were followers of orthodox Christianity. Just over one quarter (26.9\%; $\mathrm{n}=293$ ) of the study subjects enrolled in HIV care lived outside the city of Gondar (Table 1).

When we compared cases with controls, the age distribution $(\mathrm{p} \leq 0.001)$, marital status $(\mathrm{p}=0.013)$ and educational status $(\mathrm{p}=0.010)$ were significantly different in cases and controls. A higher proportion of LTFU individuals (22.9\%) were younger (age 15-24) when compared to those who were in care (12.8\%). Higher proportions of LTFU individuals had never been married (21.2\%) or were divorced or separated $(32.2 \%)$ compared to those who were in care (15.6\% and $27.8 \%$, respectively). Moreover, a higher proportion of LTFU individuals had less education (no education, 38.6\%; primary level education, 29.5\%) compared to those who were in care (33.6\% and $24.9 \%$, respectively) (Table 1$)$.

\section{Baseline clinical characteristics and chronic care follow-up status}

Among the 1076 patients who had their baseline CD4 cell count documented, 56.8\% $(\mathrm{n}=611)$ had CD4 count $\leq 200 \mathrm{cell} / \mu \mathrm{l}$ and $20.0 \%(\mathrm{n}=215)$ had a CD4 count $>350$ cell $/ \mu$ l. In contrast, among the 1070 charts that had

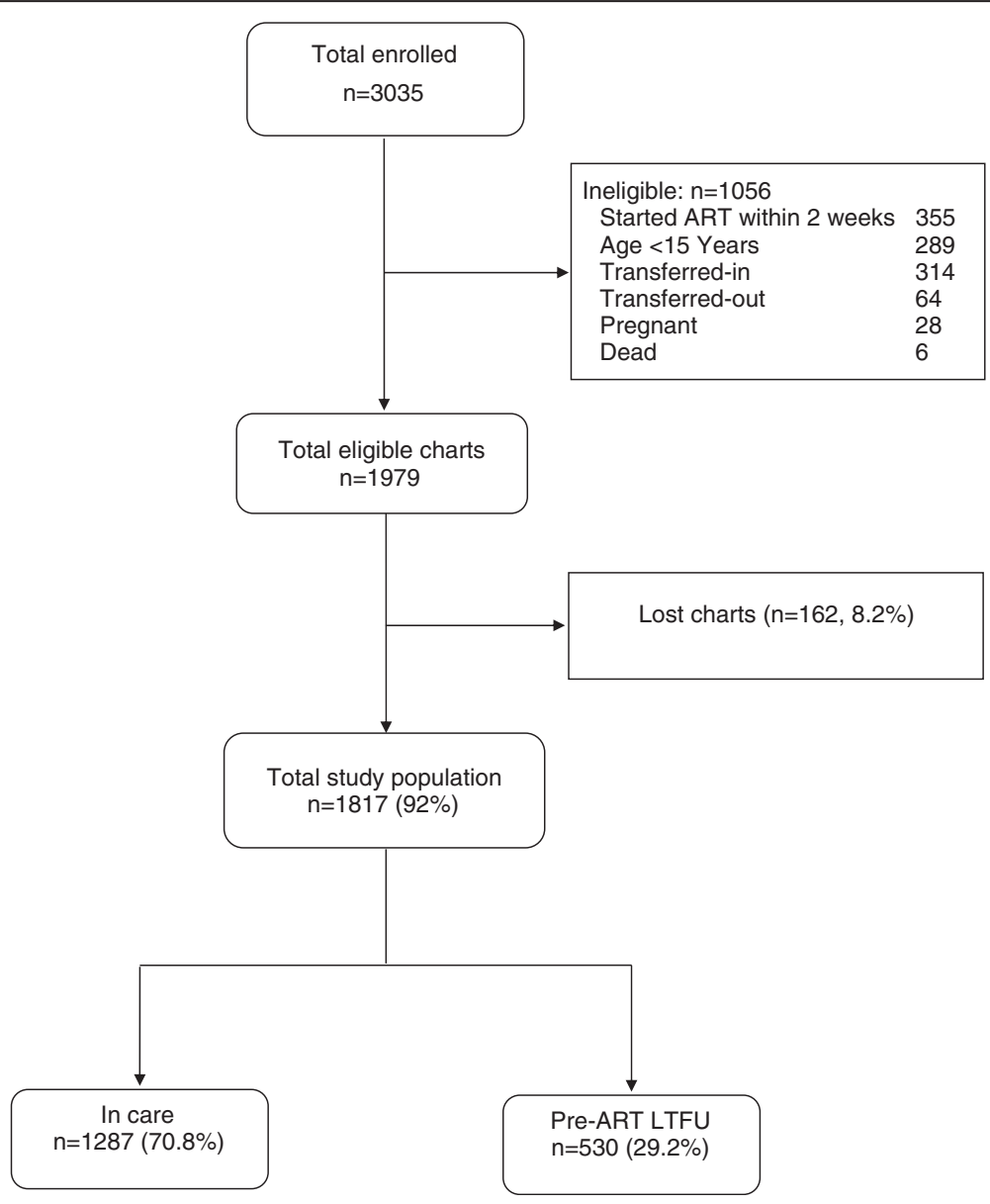

Figure 1 Profiles of HIV clients at GUH (September 11, 2008 to May 8, 2011). 
Table 1 Baseline socio-demographic characteristics of pre-ART patients at GUH (September 11, 2008 to May 8, 2011)

\begin{tabular}{|c|c|c|c|c|}
\hline Variables & Total, n (\%) & In care, n (\%) & LTFU, n (\%) & P-value \\
\hline Gender ( $N=1089)$ & & & & 0.930 \\
\hline Male & $439(40.3)$ & $292(40.2)$ & $147(40.5)$ & \\
\hline Female & $650(59.7)$ & $434(59.8)$ & $216(59.5)$ & \\
\hline Age group ( $N=1089$ ) & & & & $<0.001$ \\
\hline $15-24$ & $176(16.2)$ & $93(12.8)$ & $83(22.9)$ & \\
\hline $25-34$ & $498(45.7)$ & $327(45.0)$ & $171(47.1)$ & \\
\hline $35-44$ & $283(26.0)$ & $207(28.5)$ & $76(20.9)$ & \\
\hline $44+$ & $132(12.1)$ & 99 (13.6) & $33(9.1)$ & \\
\hline Marital status $(\mathrm{N}=1089)$ & & & & 0.013 \\
\hline Never married & $190(17.4)$ & $113(15.6)$ & $77(21.2)$ & \\
\hline Married & $498(45.7)$ & $351(48.3)$ & $147(40.5)$ & \\
\hline Divorced/separated & $319(29.3)$ & $202(27.8)$ & $117(32.2)$ & \\
\hline Widow/er & $82(7.5)$ & $60(8.3)$ & $22(6.1)$ & \\
\hline Educational status ( $\mathrm{N}=1089$ ) & & & & 0.010 \\
\hline No education & $384(35.3)$ & $244(33.6)$ & $140(38.6)$ & \\
\hline Primary & $288(26.4)$ & $181(24.9)$ & $107(29.5)$ & \\
\hline Secondary \& above & $417(38.3)$ & $301(41.5)$ & $116(32.0)$ & \\
\hline Religion ( $N=1089$ ) & & & & 0.131 \\
\hline Muslim & $87(8.0)$ & $55(7.6)$ & $32(8.8)$ & \\
\hline Orthodox & $991(91.0)$ & $661(91.0)$ & $330(90.9)$ & \\
\hline Others & $11(1.0)$ & $10(1.4)$ & $1(.3)$ & \\
\hline Place of residence $(\mathrm{N}=1089)$ & & & & 0.411 \\
\hline Gondar & $796(73.1)$ & $525(72.3)$ & $271(74.7)$ & \\
\hline Out of Gondar & $293(26.9)$ & $201(27.7)$ & $92(25.3)$ & \\
\hline Employment status ( $\mathrm{N}=995)$ & & & & 0.186 \\
\hline Employed & $568(57.1)$ & $382(58.6)$ & $186(54.2)$ & \\
\hline Unemployed & $427(42.9)$ & $270(41.4)$ & $157(45.8)$ & \\
\hline Availability of emergency contact person ( $N=1089)$ & & & & 0.396 \\
\hline Yes & $955(87.7)$ & $641(88.3)$ & $314(86.5)$ & \\
\hline No & 134 (12.3) & $85(11.7)$ & 49 (13.5) & \\
\hline
\end{tabular}

complete information about WHO staging, the majority (41.7\%; $\mathrm{n}=446)$ of patients were stage III followed by $24 \%(\mathrm{n}=257)$ stage I patients. Among 1044 patient charts that had documentation on past history of tuberculosis, $21.3 \%(n=222)$ patients had a history of tuberculosis treatment.

The distribution of CD4 cell count categories, WHO stage and recorded next appointment date were significantly different in cases versus controls $(p \leq 0.001)$. A higher proportion of LTFU patients (42.2\%) had a CD4 count $>350 \mathrm{cell} / \mu \mathrm{l}$ when compared to those who were in care (9.1\%). Similarly, higher proportions of LTFU patients (34\%) were in WHO stage I compared to those who were in care (19.1\%). Individuals who were in care $(97.8 \%)$ were more likely to have their next appointment date recorded than those that were LTFU (20.4\%) (Table 2).
Concerning the length of follow-up time in HIV chronic care, most $(61.7 \% ; n=672)$ of the patients had less than one month follow-up during their pre-ART period. The median length of time in the pre-ART period was less than one month, ranging from less than one up to 37 months. Out of a total of 363 pre-ART LTFU cases, $86 \%(n=312)$ had less than one month of follow-up, $10.4 \% \quad(n=38)$ had between one and six months of follow-up, and 3.6\% $(n=13)$ had more than seven months of follow-up time in the clinic. When we examine the time of LTFU of the 363 pre-ART LTFU patients, $84.8 \%(n=308)$ of LTFU patients failed to return since their first clinic visit or did not show up after the date of enrollment.

Regarding the practice of clinicians on documenting the next appointment date of patients in pre-ART care, the 
Table 2 Baseline clinical characteristics of pre-ART patients at GUH (September 11, 2008 to May 8, 2011)

\begin{tabular}{|c|c|c|c|c|}
\hline Variables & Total, n (\%) & In care, $\mathrm{n}(\%)$ & LTFU, n (\%) & P-value \\
\hline Patient referral information $(\mathrm{N}=1081)$ & & & & 0.118 \\
\hline Within the hospital & $796(73.6)$ & $518(72.1)$ & $278(76.6)$ & \\
\hline Outside the hospital & $285(26.4)$ & $200(27.9)$ & $85(23.4)$ & \\
\hline Disclosure of HIV+ status ( $\mathrm{N}=1089)$ & & & & 0.174 \\
\hline To spouse/family members only & $616(56.6)$ & $423(58.3)$ & $193(53.2)$ & \\
\hline To relatives/friends only & $86(7.9)$ & $61(8.4)$ & $25(6.9)$ & \\
\hline To spouse/family members \& relatives/friends & $35(3.2)$ & $23(3.2)$ & $12(3.3)$ & \\
\hline To no one & $352(32.3)$ & $219(30.2)$ & $133(36.6)$ & \\
\hline CD4 cell count/ $\mu \mathrm{l}(\mathrm{N}=1076)$ & & & & $<0.001$ \\
\hline$\leq 200$ & $611(56.8)$ & $505(69.8)$ & $106(30.0)$ & \\
\hline $201-250$ & $111(10.3)$ & $73(10.1)$ & $38(10.8)$ & \\
\hline $251-300$ & $75(7.0)$ & $50(6.9)$ & $25(7.1)$ & \\
\hline $301-350$ & $64(5.9)$ & $29(4.0)$ & $35(9.9)$ & \\
\hline$>350$ & $215(20.0)$ & $66(9.1)$ & $149(42.2)$ & \\
\hline WHO stage $(\mathrm{N}=1070)$ & & & & $<0.001$ \\
\hline Stage I & $257(24.0)$ & $137(19.1)$ & $120(34.0)$ & \\
\hline Stage II & $194(18.1)$ & $126(17.6)$ & $68(19.3)$ & \\
\hline Stage III & $446(41.7)$ & $332(46.3)$ & $114(32.3)$ & \\
\hline Stage IV & $173(16.2)$ & $122(17.0)$ & $51(14.4)$ & \\
\hline History of TB treatment $(\mathrm{N}=1044)$ & & & & 0.058 \\
\hline Yes & $222(21.3)$ & $159(23.0)$ & $63(17.9)$ & \\
\hline No & $822(78.7)$ & $533(77.0)$ & $289(82.1)$ & \\
\hline Next appointment recorded $(\mathrm{N}=1089)$ & & & & $<0.001$ \\
\hline Yes & $784(72.0)$ & $710(97.8)$ & $74(20.4)$ & \\
\hline No & $305(28.0)$ & $16(2.2)$ & 289 (79.6) & \\
\hline
\end{tabular}

next appointment date was not documented on patients' medical record for more than a quarter $(28 \%$; $=305)$ of patients (Table 2).

\section{Socio-demographic and clinical factors associated with pre-ART LTFU}

After controlling for the effects of gender, age, marital status, educational status, place of residence, HIV disclosure, history of tuberculosis treatment, CD4 cell count, WHO stage, and record of next appointment, five variables were found to be associated significantly with pre-ART LTFU. As shown in Table 3, males had twice the odds of being LTFU during the pre-ART period compared to females (adjusted OR $=2.00$ (95\% CI: 1.15, 3.46)). Married patients had a $61 \%$ reduced odds of being LTFU during the pre-ART period compared to those who had never been married (adjusted OR $=0.39(95 \%$ CI: 0.19, 0.79)). Compared to patients with a baseline CD4 count $\leq 200$ cells/ $\mu$ l, the adjusted odds ratio for being LTFU before starting ART was 2.64 (95\% CI: 1.05, 6.65), 5.21 (95\% CI: 1.94, 13.99), and 12.10 (95\% CI:
$6.33,23.12)$ for patients with a CD4 count of 251-300 cells $/ \mu \mathrm{l}, 301-350$ cells $/ \mu \mathrm{l}$, and $>350$ cells $/ \mu \mathrm{l}$ respectively. Similarly, patients who were in WHO stage I at baseline, had an adjusted odds ratio for LTFU during the preART period of 2.81 (95\% CI: 1.15, 6.91) when compared to patients who were in WHO stage IV.

Patients whose next appointment date was not documented on their chart had substantially increased odds of being LTFU during the pre-ART period compared to those who had a record of the next appointment date documented (adjusted OR = 241.39 (95\% CI: 119.90, 485.97).

\section{Discussion}

This study is one of the few reports from a resource limited-setting to describe the factors associated with LTFU among a pre-ART population of PLHIV. This group of patients has not been well studied compared to PLHIV who are receiving ART. This is despite emerging evidence of the poor disease outcomes in this pre-ART group. This highlights the need for greater focus upon retention of pre-ART patients from the beginning of HIV care, and not 
Table 3 Socio-demographic and clinical factors associated with pre-ART loss to follow-up at GUH

\begin{tabular}{|c|c|c|c|c|c|c|}
\hline \multirow[b]{2}{*}{ Variables } & \multirow[b]{2}{*}{ n total } & \multicolumn{3}{|c|}{ Crude OR $(95 \% \mathrm{Cl})$} & \multicolumn{2}{|c|}{ Adjusted $^{+}$OR $(95 \% \mathrm{Cl})$} \\
\hline & & n (\%) & OR & $95 \% \mathrm{Cl}$ & $\overline{\mathrm{OR}}$ & $95 \% \mathrm{Cl}$ \\
\hline Gender & 1089 & & & & & \\
\hline Male & & $439(40.3)$ & 1.01 & $(0.78,1.31)$ & 2.00 & $(1.15,3.46)^{*}$ \\
\hline Female & & $650(59.7)$ & 1.00 & & 1.00 & \\
\hline Age group & 1089 & & & & & \\
\hline $15-24$ & & $176(16.2)$ & 1.00 & & 1.00 & \\
\hline $25-34$ & & $498(45.7)$ & 0.59 & $(0.41,0.83)^{*}$ & 0.80 & $(0.40,1.61)$ \\
\hline $35-44$ & & $283(26.0)$ & 0.41 & $(0.28,0.61)^{*}$ & 0.63 & $(0.28,1.41)$ \\
\hline $44+$ & & $132(12.1)$ & 0.37 & $(0.23,0.61)^{*}$ & 0.77 & $(0.30,2.00)$ \\
\hline Marital status & 1089 & & & & & \\
\hline Never married & & $190(17.4)$ & 1.00 & & 1.00 & \\
\hline Married & & $498(45.7)$ & 0.62 & $(0.43,0.87)^{*}$ & 0.39 & $(0.19,0.79)^{*}$ \\
\hline Divorced/separated & & $319(29.3)$ & 0.85 & $(0.59,1.23)$ & 1.18 & $(0.57,2.44)$ \\
\hline Widow/er & & $82(7.5)$ & 0.54 & $(0.31,0.95)^{*}$ & 0.54 & $(0.18,1.68)$ \\
\hline Educational status & 1089 & & & & & \\
\hline No education & & $384(35.3)$ & 1.00 & & 1.00 & \\
\hline Primary & & $288(26.4)$ & 1.03 & $(0.75,1.41)$ & 1.47 & $(0.78,2.77)$ \\
\hline Secondary \& above & & $417(38.3)$ & 0.67 & $(0.50,0.91)^{*}$ & 0.88 & $(0.48,1.63)$ \\
\hline Disclosure of HIV+ status & 1089 & & & & & \\
\hline To spouse/family members only & & $616(56.6)$ & 1.00 & & 1.00 & \\
\hline To relatives/friends only & & $86(7.9)$ & 0.90 & $(0.55,1.47)$ & 0.38 & $(0.12,1.19)$ \\
\hline To spouse/family members \& relatives/friends & & $35(3.2)$ & 1.14 & $(0.56,2.35)$ & 1.47 & $(0.41,5.32)$ \\
\hline To no one & & $352(32.3)$ & 1.33 & $(1.01,1.75)^{*}$ & 0.65 & $(0.37,1.14)$ \\
\hline Place of residence & 1089 & & & & & \\
\hline Gondar & & $796(73.1)$ & 1.00 & & 1.00 & \\
\hline Out of Gondar & & $293(26.9)$ & 0.89 & $(0.67,1.18)$ & 1.07 & $(0.61,1.87)$ \\
\hline History of TB treatment & 1044 & & & & & \\
\hline Yes & & $222(21.3)$ & 1.00 & & 1.00 & \\
\hline No & & $822(78.7)$ & 1.37 & $(0.99,1.89)$ & 0.73 & $(0.39,1.35)$ \\
\hline CD4 cell count/ $\mu \mathrm{l}$ & 1076 & & & & & \\
\hline$\leq 200$ & & $611(56.8)$ & 1.00 & & 1.00 & \\
\hline $201-250$ & & $111(10.3)$ & 2.48 & $(1.59,3.87)^{*}$ & 2.22 & $(0.95,5.19)$ \\
\hline $251-300$ & & $75(7.0)$ & 2.38 & $(1.41,4.02)^{*}$ & 2.64 & $(1.05,6.65)^{*}$ \\
\hline $301-350$ & & $64(5.9)$ & 5.75 & $(3.37,9.82)^{*}$ & 5.21 & $(1.94,13.99)^{*}$ \\
\hline$>350$ & & $215(20.0)$ & 10.76 & $(7.52,15.38)^{*}$ & 12.10 & $(6.33,23.12)^{*}$ \\
\hline WHO stage & 1070 & & & & & \\
\hline Stage I & & $257(24.0)$ & 2.10 & $(1.39,3.15)^{*}$ & 2.81 & $(1.15,6.91)^{*}$ \\
\hline Stage II & & $194(18.1)$ & 1.29 & $(0.83,2.01)$ & 1.75 & $(0.69,4.45)$ \\
\hline Stage III & & $446(41.7)$ & 0.82 & $(0.56,1.21)$ & 1.72 & $(0.77,3.81)$ \\
\hline Stage IV & & $173(16.2)$ & 1.00 & & 1.00 & \\
\hline Next appointment record & 1089 & & & & & \\
\hline Yes & & $784(72.0)$ & 1.00 & & 1.00 & \\
\hline No & & $305(28.0)$ & 173.30 & $(99.25,302.61)^{*}$ & 241.39 & $(119.90,485.97)^{*}$ \\
\hline
\end{tabular}

OR: Odds Ratio, Cl: Confidence Interval, ${ }^{*} \mathrm{p} \leq 0.05,{ }^{+} \mathrm{A}$ total of 1015 subjects included in the adjusted model. 
just after being initiated on ART [5,10,11]. Our study has examined the socio-demographic and clinical factors associated with LTFU during the pre-ART period in Ethiopia and found that male gender, being unmarried, CD4 cell count $>250$ cells $/ \mu \mathrm{l}$, WHO stage I, and not having documented next appointment date on the medical chart were all associated significantly with increased odds of pre-ART LTFU.

Similar with a study conducted in southern Ethiopia [5], the majority of patients in this study started care after reaching compromised immune system. This was shown by the high proportion of patients that had baseline CD4 count of $\leq 200$ cells/ $\mu$ l and WHO stage III and IV. In this study males were found to be at higher risk of LTFU before starting ART. This finding was consistent with studies conducted in Uganda [17] and Malawi [14]. Unlike the findings of previous studies, this study identified an association between marital status and pre-ART LTFU. In this study being married was associated with lower odds of pre-ART LTFU compared to those who had never married. This could be explained by the importance of family support in HIV care $[18,19]$.

Low CD4 cell count was identified as an important risk factor for pre-ART LTFU at a semi-private hospital in Durban, South Africa [16] and in a non-governmental clinic in Jinja, Uganda [17]. In studies conducted in Malawi and Kenya, having an advanced WHO stage of illness (III and IV) was identified as a risk factor for preART LTFU [14]. Our study findings contradicted this, with increased odds of LTFU among patients with better immune system indicators (higher CD4 cell count and less advanced WHO stage), and this could be due to methodological differences with respect to operational definitions and also differences between the study settings. Unlike our study, which defined preART patients as all newly enrolled HIV patients who did not start ART, studies conducted in South Africa [16], Uganda [17], Malawi and Kenya [14] focused on pre-ART patients with low CD4 cell count and advanced WHO stage. These groups of patient are not representative of all pre-ART patients who are enrolled in HIV chronic care and expected to have periodic follow-up visits. On the other hand, our finding regarding the association between less advanced disease stage and pre-ART LTFU was consistent with a study conducted in southern Ethiopia that employed a similar operational definition and study settings [5]. Furthermore, we found that the risk of pre-ART LTFU increased progressively with increasing CD4 cell count, indicating a dose response relationship. This finding was supported by the study conducted in South Africa where the probability of returning to care dropped substantially for patients with higher CD4 cell count [10]. Part of the explanation for the increased odds of pre-
ART LTFU among patients with better immune status or less advanced disease stage could result from patients' self-assessment of feeling healthy and, therefore, not in need of health care [20].

The other contributory factor to pre-ART LTFU identified in this study was lack of documentation of patients' next appointment date by clinicians. Studies conducted in Malawi and Kenya also identified patients' charts that did not have the next appointment date specified, but excluded them from the analysis [14]. We included these individuals without recorded appointment date because lack of documentation itself might be indicative of the type of service patients received about specific follow-up visit days and could have an effect on patients' follow-up. Indeed, this study found that patients without a record of the next visit date had a high chance of being LTFU during the pre-ART period. This could be because a clinician who failed to record the next appointment date on the patient's chart might also omit to provide written or verbal information for the patient about the specific date of the next visit.

The main limitation of the study was the lack of or missing data regarding baseline socio-demographic and clinical variables and also our inability to locate some of the patient charts during the data collection period. Charts with no baseline information on the intake form, especially demographic data, and chart numbers that could not be found during random sampling were replaced by the next study subject (number) in the list of the study population. The rate of replacement of samples was low (5.5\%). The other limitation was the study setting that was confined to only one university hospital, and hence the result might not be extrapolated to other locations.

\section{Conclusions}

Our study found that patients who were less ill were more likely to be LTFU during the pre-ART period. The risk of pre-ART LTFU increased progressively with increasing CD4 cell count. These findings highlight the importance of giving greater attention to the care of preART patients, starting from the time that they learn of their HIV positive serostatus. The standard of care for pre-ART patients needs to be revised to include measures to encourage follow-up, including early initiation of ART. Efforts to prevent pre-ART LTFU should be strengthened through mechanisms that proactively indentify and manage pre-ART patients who are likely to be non-adherent to care and become LTFU. There should be standardized definition of pre-treatment LTFU and appointment system that can monitor and easily flag-out missed appointment patients. Intensive adherence counseling and adequate information should be given to patients at-risk of LTFU at each visit especially 
during the first visit. The completeness of the medical records, the standard of record keeping and obstacles to retrieving charts also indicate a serious problem that needs due attention from clinicians and data personnel.

\section{Abbreviations}

AIDS: Acquired Immune Deficiency Syndrome; ART: Anti-Retroviral Treatment; GUH: Gondar University Hospital; HIV: Human Immune-deficiency Virus; LTFU: Loss to Follow-up; PLHIV: People Living with HIV/AIDS; Pre-ART: Pre-Antiretroviral Treatment; WHO: World Health Organization.

\section{Competing interests}

The authors declare that they have no competing interests.

\section{Authors' contributions}

IA had primary responsibility in the process of conceptualization and research design, data collection, data analysis and interpretation, and manuscript drafting and revision. STG and SL assisted in the conceptualization and design of the study, development of data collection instruments, interpretation of the results, and preparation of the manuscript MD participated in the preparation of the manuscript and revising it critically for important intellectual content. All authors read and approved the final manuscript.

\section{Authors' information}

IA: RN, MPH; Care and Support Advisor at International Training and Education Center for Health-Ethiopia. He has expertise in HIV/AIDS treatment, care and support. STG: MPH; Research Scientist at University of Washington, Department of Global Health. She has expertise in Epidemiology and Bio-cultural Anthropology.

SL: MPH in Epidemiology; Researcher and Instructor at Addis Continental Institute of Public Health. MD: MD, MPH, PhD; Associate Professor at Addis Continental Institute of Public Health, Department of Public Health. She has expertise in Tuberculosis and HIV/AIDS Programs.

\section{Acknowledgements}

We thank GUH for facilitating conditions while conducting the study. We would like to extend our heartfelt thanks and appreciation to supervisors and data collectors (ART clinic Nurses, adherence case managers and adherence supporters). Our special appreciation goes to Visions for Development, Inc. for its funding to cover some of the costs related to data collection.

\section{Author details}

${ }^{1}$ University of Gondar and Addis Continental Institute of Public Health, P.O. Box 180432, Addis Ababa, Ethiopia. ${ }^{2}$ Department of Global Health, University of Washington, 901 Boren Avenue, Suite 1100, Seattle, Washington 98104, USA. ${ }^{3}$ Addis Continental Institute of Public Health, P.O. Box 26751/ 1000, Addis Ababa, Ethiopia.

Received: 20 November 2012 Accepted: 11 September 2013 Published: 22 September 2013

\section{References}

1. United Joint United Nations Programme on HIV/AIDS: UNAIDS Report on the Global AIDS Epidemic. Geneva: UNAIDS; 2010

2. EHNRI FMoH: HIV Related Estimates and Projections for Ethiopia. Addis Ababa: $\mathrm{FMoH} ; 2012: 6-14$.

3. Federal HIV/AIDS Prevention and Control Office FMoH: Guidelines for Implementation of HIV/AIDS Case Management in Ethiopia. Addis Ababa: FHAPCO; 2009.

4. Federal HIV/AIDS Prevention and Control Office FMoH: Multi-sectoral response to HIV/AIDS in Ethiopia 2011 Fiscal Year Annual Report. Addis Ababa: FHAPCO; 2011.

5. Mulissa Z, Jerene $D$, Lindtiørn B: Patients present earlier and survival has improved, but pre-art attrition is high in a six-year HIV cohort data from Ethiopia. PLOS ONE 2010, 5:e13268.

6. Jerene D, Lindtjørn B: Disease progression among untreated HIV-infected patients in South Ethiopia: implications for patient care. Med Gen Med 2005, 7:66.
7. Bassett IV, Regan S, Chetty S, Giddy J, Uhler LM, Holst H, Ross D, Katze JN, Walenskya RP, Freedberga KA, Losina E: Who starts antiretroviral therapy in Durban, South Africa?... not everyone who should. AIDS 2010, 24:S37-S44.

8. Raguenaud M-E, Isaakidis P, Zachariah R, Te V, Soeung S, Akao K, Kumar V: Excellent outcomes among HIV+ children on ART, but unacceptably high pre-ART mortality and losses to follow-up: a cohort study from Cambodia. BioMed Central 2009, 9:54.

9. Thai S, Koole O, Un P, Ros S, Munter PD, Damme WV, Jacques G, Colebunders R, Lynen L: Five-year experience with scaling-up access to antiretroviral treatment in an HIV care programme in Cambodia. Trop Med Int Health 2009, 14:1048-1058.

10. Larson BA, Brennan A, McNamara L, Long L, Rosen S, Sanne I, Fox MP: Early loss to follow up after enrolment in pre-ART care at a large public clinic in Johannesburg, South Africa. Trop Med Int Health 2010, 15:43-47.

11. Losina E, Bassett IV, Giddy J, Chetty S, Regan S, Walensky RP, Ross D, Scott CA, Uhler LM, Katz JN, et al: The "ART" of linkage: pre-treatment loss to care after HIV diagnosis at two PEPFAR sites in Durban, South Africa. PLOS ONE 2010, 5:e9538.

12. Ahmed I, Gugsa S, Mengiste D, Mohammed Y, Petracca F: A Proactive Approach to Retain Clients on Treatment and Care in Ethiopia. In XVII International AIDS Conference held at Mexico City. 2008.

13. Federal HIV/AIDS Prevention and Control Office FMoH: Guidelines for management of opportunistic infections and antiretroviral treatment in adolescents and adults in Ethiopia. Addis Ababa: FHAPCO; 2008.

14. Zachariaha R, Tayler-Smith K, Manzi M, Massaquoi M, Mwagomba B, Griensven J, Engelgem I, Arnould L, Schouten EJ, Chimbwandira FM, Harries AD: Retention and attrition during the preparation phase and after start of antiretroviral treatment in Thyolo, Malawi, and Kibera, Kenya: implications for programmes? Trans R Soc Trop Med Hyg 2011, 4191:10.

15. Maskew M, MacPhail P, Menezes C, Rubel D: Lost to follow up contributing factors and challenges in South African patients on antiretroviral therapy. S Afr Med J 2007, 97:853-857.

16. Bassett IV, Wang B, Chetty S, Mazibuko M, Bearnot B, Giddy J, Lu Z, Losina E, Walensky RP, Freedberg KA: Loss to care and death before antiretroviral therapy in Durban, South Africa. Natl Inst Health 2009, 51:135-139.

17. Amuron B, Namara G, Birungi J, Nabiryo C, Levin J, Grosskurth H, Coutinho A, Jaffar S: Mortality and loss-to-follow-up during the pretreatment period in an antiretroviral therapy programme under normal health service conditions in Uganda. BioMed Central 2009, 9:290.

18. DiMatteo MR: Social support and patient adherence to medical treatment: a meta-analysis. American Psychological Association, Inc 2004 23:207-218.

19. Amberbir A, Woldemichael K, Getachew S, Girma B, Deribe K: Predictors of adherence to antiretroviral therapy among HIV-infected persons: a prospective study in Southwest Ethiopia. BMC Public Health 2008, 8:265.

20. Assefa Y, Damme W, Mariam DH, Kloos aH: Toward universal access to HIV counseling and testing and antiretroviral treatment in Ethiopia: looking beyond HIV testing and ART initiation. AIDS Patient Care STDS 2010, 24:521-525.

doi:10.1186/1471-2458-13-867

Cite this article as: Ahmed et al: Predictors of loss to follow-up before HIV treatment initiation in Northwest Ethiopia: a case control study. BMC Public Health 2013 13:867.

\section{Submit your next manuscript to BioMed Central and take full advantage of:}

- Convenient online submission

- Thorough peer review

- No space constraints or color figure charges

- Immediate publication on acceptance

- Inclusion in PubMed, CAS, Scopus and Google Scholar

- Research which is freely available for redistribution 